\title{
SMALL ANGLE NEUTRON SCATTERING STUDIES OF CRITICAL PHENOMENA IN A THREE-COMPONENT MICROEMULSION
}

H. Seto, G. D. Wignall*, R. Triolo**, D. Chillura-Martino** and S. Komura

Faculty of Integrated Arts and Sciences, Hiroshima University, Japan

*Solid State Division, Oak Ridge National Laboratory, USA

* Dipartimento de Chimica Fisica, University of Palermo, Italy

short running title: SANS studies of critical phenomena in a microemulsion

keywords:small angle neutron scattering, critical phenomena, crossover, microemulsion address for proofs:

\section{Dr. Hideki Seto}

Faculty of Integrated Arts and Sciences,

Hiroshima University

1-7-1 Kagamiyama, Higashihiroshima 739, Japan

Sulmitted to the proceedings of

"The International Symposium on Colloids and Polymer Science", Oct 10-13, 1996. Nagoya.

\section{DISTRIBUTION OF THIS DOCIMENT IS UNLIMTED}




\section{DISCLAIMER}

Portions of this document may be illegible in electronic image products. Images are produced from the best available original document. 


\begin{abstract}
Critical density fluctuations of a "water-in-oil" microemulsion consisting of water, benzene, and BHDC (benzyldimethyl-n-hexadecyl ammonium chloride) have been observed near the phase boundary by a small angle neutron scattering (SANS). The observed profiles were well described by the product of a form factor of spherical droplets and a structure factor, consisting of a term describing the inter-droplet correlations and also an Omstein-Zernike component describing the droplet density fluctuations. Allowance was also made for the droplet polydispersity, though the width of the distribution turned out to be very small $(1-2 \%)$. The observed temperature dependence of the osmotic compressibility was fitted using the crossover function proposed by Belyakov et al., and the Ginzburg numbers were obtained of the order of 10-2. These results indicate that long range interdroplet forces are not significant in this system, which displays upper critical solution temperature (UCST) behavior. In contrast, previous studies of systems displaying with lower critical solution temperature (LCST) behavior [e. g. water, n-decane and AOT (dioctyl sulfosuccinate sodium salt)] indicate that long range interactions appear to dominate the phase separation behavior.
\end{abstract}


The critical behavior of density fluctuations in microemulsions with a droplet structure can be treated analogously to simple fluids, because the radius is virtually constant throughout the phase separation and the droplet density may be regarded as an order parameter. Because of the nature of the droplet systems, its critical behavior is expected to belong to the 3D Ising universality class. However, the observed critical exponents do not always coincide with the exact values of the 3D Ising model. In particular, the well-known ternary system (WDA), consisting of an oil-rich mixture of Water, $n$-Decane, and AOT (dioctyl sulfosuccinate sodium salt) has been the subject of a range of experimental investigations. [1 - 4] This system is homogeneous at room temperature and decomposes as the temperature is increased, thus exhibiting critical phenomena below the decomposition temperature, i. e. the phase diagram show an LCST transition. The various experimental studies performed on this system gave values of the critical exponents, $\gamma$ and $\nu$, which did not coincide with either the 3D-Ising or Fisher's renormalized 3D-Ising values.

Alternatively, Kiselev and his coworkers $[5,6]$ have proposed an analytical form of the susceptibility, which describes the fluctuations of the order parameter over the whole temperature range in the mixed state of the system,

$\hat{\tau}=\left(1+2.333 S_{0}^{\Delta / \gamma}\right)^{(\gamma-1) / \Delta}\left[S_{0}^{-1}+\left(1+2.333 S_{0}^{\Delta / \gamma}\right)^{-\gamma / \Delta}\right]$

where $S_{0}=S_{0} G i / C_{\mathrm{MF}}$ and $\hat{\tau}=\tau / G i$ are a renormalized susceptibility and temperature. $S_{0}, G i, C_{\mathrm{MF}}$, and $\tau=\left|T^{-1}-T_{\mathrm{c}}^{-1}\right| / T_{\mathrm{c}}^{-1}$ represent the susceptibility of the order parameter fluctuation, the Ginzburg number (i.e. the value of reduced temperature separating the mean field and 3D-Ising regions), the critical amplitude of the mean field approximation and the reduced temperature, respectively. This theory has the advantage that it may be applied quite generally over the whole crossover regime including the scaling limits both very near and very far from the critical point. Seto et al. [7] have recently used this approach to interpret SANS data from WDA microemulsions, and showed that the critical behavior can be explained by a crossover from the mean field to $3 \mathrm{D}$ Ising regimes. The Ginzburg number was found to be of the order of $10^{-3}$, which is an order of magnitude smaller than 
in simple fluids. This finding suggests that the dominant interactions underlying the phase separation are long-ranged. In this system, three types of interactions between droplets are known; a hard core repulsive force, a hydrophobic attraction, and an electrostatic interaction originating in the charge fluctuations. [8] Among these interactions, the electrostatic one should control the phase separation phenomenon, because rather strong attractive forces are required to produce LCST behavior, as the system decomposes as the temperature is raised and the entropy increases. This hypothesis is consistent with the small value of the Ginzburg number because the electrostatic interaction must be long-ranged.

In order to clarify the relation between the phase behavior, interactions between droplets, and the Ginzburg number, we have undertaken further SANS studies of critical phenomenon in a different three-component microemulsion system called WBB, consisting of Water, Benzene, and BHDC (benzyldimethyl-n-hexadecyl ammonium chloride). This system also has a water-in-oil type droplet structure at room temperature and decomposes with decreasing temperature. Above the (UCST) phase separation point, critical phenomena have been investigated by Beysens and coworkers $[9,10]$, who obtained the critical indexes, $\gamma=1.18$ and $\nu=0.60$, and concluded that their data could be interpreted within the 3D-Ising universality. However, Fisher's renormalized critical exponents were not obtained.

The current SANS experiments were performed at 30m-SANS facility at Oak Ridge National Laboratory with a wave length of $0.4750 \mathrm{~nm}$ and sample-detector distance of $14 \mathrm{~m}$ and $5 \mathrm{~m}$, which gave a range of momentum transfer of $0.047 \leq Q \leq 1.2\left[\mathrm{~nm}^{-1}\right]$. Quartz cells (1 mm path length) were used to contain the samples, whose temperature was controlled by a circulation bath system with an accuracy of $\pm 0.2 \mathrm{~K}$. The chemicals, BHDC, benzene, $\mathrm{D}_{2} \mathrm{O}$, and $\mathrm{H}_{2} \mathrm{O}$ were purchased from Tokyo Kasei Co. Ltd., Mallinckrodt Inc., Aldrich Chem. and J. T. Baker Inc. It is not known whether the phase transition may be perturbed by isotope effects, due to $\mathrm{H}_{2} \mathrm{O}$ with $\mathrm{D}_{2} \mathrm{O}$, so in order to minimize this possibility, we prepared four mixtures where the volume fraction of each composition is almost the same as the critical composition for pure $\mathrm{H}_{2} \mathrm{O}$, i. e., mass ratios of water to BHDC were 2.026 
and 2.005 for the $60 \% \mathrm{D}_{2} \mathrm{O}$ and $50 \% \mathrm{D}_{2} \mathrm{O}$ samples, respectively. The mass fractions of benzene with respect to the total mass $\left(C_{\mathrm{B}}\right)$ were 0.91 and 0.92 respectively (see Table $I$ ).

In order to fit the observed SANS profiles, we assumed a scattering function which is the product of an intraparticle form factor, $P(Q)$, and an interparticle structure factor, $S(Q)$, with a constant background term $I_{\mathrm{B}}$. An analytical form for the Schultz size distribution proposed by Kotlarchyk and Chen [11] was used to model possible polydispersity effects. Thus, the fitting parameters are the scattering density difference between the droplet and the solvent $(\Delta Q)$, the mean radius of droplet $(R)$, and width parameter of polydispersity $(Z)$. We assumed that $S(Q)$ can be represented as the sum of an interdroplet component, $S_{\mathrm{ID}}(Q)$, and an Omstein-Zernike structure factor $S_{0 z}(Q)$ describing the droplet density fluctuation. As the interdroplet structure factor, a single Gaussian function was used.

$S(Q)=1+S_{\mathrm{ID}}(Q)+S_{\mathrm{OZ}}(Q), S_{\mathrm{OZ}}(Q)=S_{0} /\left(1+\xi^{2} Q^{2}\right), S_{\mathrm{ID}}(Q)=S_{0} \mathrm{G} \exp \left(-\left(Q-Q_{0}\right)^{2} / \sigma^{2}\right)$

The fitting parameters obtained for each samples are shown in Table II. Apart from the parameters of $S_{\mathrm{OZ}}(\mathrm{Q})$, there was little variation with temperature in one phase region. The temperature variation of the susceptibility for the droplet density fluctuation $S_{0}$ was obtained and fitted to the crossover function (eq. 1) for the case of 3D-Ising model, (i. e., $\gamma=1.26$ and $\Delta=0.51$ ). The experimental observations were well described by the function and resultant fitting parameters, $T_{\mathrm{s}}$ (in this case, a spinodal temperature was obtained in spite of $T_{\mathrm{c}}$, because the samples were not the critical concentration), $G i$, and $C_{\mathrm{MF}}$, are shown in Table III. The values of Ginzburg number are of the order of $10^{-2}$, which is an order of magnitude greater than in the case of WDA and is of the same order as in simple fluids.

The polydispersity was estimated from the observed values of $Z$ and was very small (i. e. a few percent). The value of $Q_{0}$ indicates that an interdroplet correlation exists with a spacing of about $20 \mathrm{~nm}$. From these results, we conclude that the system consists of relatively monodisperse droplets 
(radius $-5 \mathrm{~nm}$ ), which scatter uniformly. Therefore, the general features of this system are quite similar to those of a simple fluid and may be expected to exhibit 3D-Ising critical behavior.

In Fig. 1, the renormalized susceptibilities are plotted versus the renormalized temperature. The data points fall in the intermediate region between the pure-Ising and the crossover regions, in contrast to the WDA system, which stayed in the crossover region. [7] This behavior is reflected in the values of $G i$ for the WDA and WBB; the former being more mean field-like, while the latter is more Ising-like. As discussed above, mean field behavior is expected when long-ranged interactions are dominant in the phase separation phenomenon. The LCST type phase diagram of the WDA system suggests that a temperature dependent attractive force drives the phase separation behavior. However, the UCST type transitions (as observed in the WBB system) do not require such an interaction. The present results from the WBB system indicate that the droplets scatter rather uniformly and the Ginzburg number is of the same order as that of simple fluids and are therefore consistent with its phase behavior.

\section{Acknowledgements}

The research at Oak Ridge was supported by the Division of Materials Sciences, U. S. Department of Energy under contract No. DE-AC05-960R22464 with Lockheed Martin Energy Research Corp., and also by the Japan-U.S. Cooperative Research Program on Neutron Scattering. 


\section{References}

[1] Huang J. S., and Kim M. W. (1982) Phys. Rev. Lett. 47: 1462.

[2] Kotlarchyk M., Chen S.-H., Huang J. S. (1983) Phys. Rev. A 28: 508.

[3] Honorat P., Roux D., and Bellocq A. M. (1984) J. Phys. (Paris) Lett. 45: L-961.

[4] Rouch J., Safouane A., Tartaglia P., and Chen S.-H. (1989) J. Chem. Phys. 90: 3756.

[5] Belyakov M. Y. and Kiselev S. B. (1992) Physica A190: 75.

[6] Anisimov M. A., Kiselev S. B., Sengers J. V., and Tang S. (1992) Physica A188, 487.

[7] Seto H., Schwahn D., Nagao M., Yokoi E., Komura S., Imai M., and Mortensen K. (1996)

Physical Review E 54: 629.

[8] Tingey J. M., Fulton J. L., and Smith R. D. (1990) J. Phys. Chem. 94: 1997.

[9] Jayalakshmi Y. and Beysens D. (1992), Physical Review A 45: 8709.

[10] Aschauer R. and Beysens D. (1993) Physical Review E 47: 1850.

[11] Kotlarchyk M. and Chen S.-H. (1983) J. Chem. Phys. 79: 2461.

The author to whom correspondence:

Hideki Seto, Ph. D.,

Faculty of Integrated Arts and Sciences,

Hiroshima University

1-7-1 Kagamiyama, Higashihiroshima 739, Japan 


\section{Figure Caption}

Fig. 1 All the observed temperature dependence of the inverse susceptibility of droplet density.

fluctuation is shown. The vertical axis indicates the inverse of the renormalized susceptibility and the horizontal the renormalized temperature. The pure Ising region is characterized by the reduced temperature which is smaller than the Ginzburg number, i. e., $\hat{\tau}=\tau / G i<1$. 
Table. I Parameters for sample preparation.

\begin{tabular}{cccc}
\hline \hline sample & $\mathrm{D}_{2} \mathrm{O}$ volume fraction(\%) & $\mathrm{M}_{\text {water }} / \mathrm{M}_{\mathrm{BHDC}}$ & $c_{\mathrm{B}}$ \\
\hline $\mathrm{C} 091$ & 60 & 2.026 & 0.91 \\
$\mathrm{C} 092$ & 60 & 2.026 & 0.92 \\
$\mathrm{C} 191$ & 50 & 2.005 & 0.91 \\
$\mathrm{C} 192$ & 50 & 2.005 & 0.92 \\
\hline \hline
\end{tabular}

Table. II The obtained fit parameters to explain the scattering intensities.

\begin{tabular}{cccccccc}
\hline \hline sample & $I_{\mathrm{B}}\left(\mathrm{cm}^{-1}\right)$ & $Z$ & $R(\mathrm{~nm})$ & $\Delta \varrho\left(10^{10} \mathrm{~cm}^{2}\right)$ & $S_{0}^{\mathrm{G}}\left(\mathrm{cm}^{-1)}\right.$ & $Q_{0}\left(\mathrm{~nm}^{-1}\right)$ & $\sigma\left(\mathrm{nm}^{-1}\right)$ \\
\hline $\mathrm{C} 091$ & 0.541 & 70.1 & 4.91 & 2.073 & 0.161 & 0.318 & 0.095 \\
$\mathrm{C} 092$ & 0.565 & 70.0 & 4.91 & 2.074 & 0.189 & 0.314 & 0.108 \\
$\mathrm{C} 191$ & 0.621 & 69.3 & 4.56 & 1.393 & 0.269 & 0.319 & 0.134 \\
$\mathrm{C} 192$ & 0.592 & 71.8 & 4.43 & 1.454 & 0.237 & 0.317 & 0.115 \\
\hline \hline
\end{tabular}

Table. III The parameters to fit the crossover function.

\begin{tabular}{lccc}
\hline sample & $T_{\mathrm{s}}(\mathrm{K})$ & Gi (units of 10-2) & $C_{\mathrm{MF}}$ (units of 10-2) \\
\hline C091 & $282.8 \pm 0.7$ & $0.68 \pm 0.48$ & $8.20 \pm 0.24$ \\
$\mathrm{C} 092$ & $281.7 \pm 1.8$ & $1.57 \pm 0.16$ & $9.58 \pm 1.62$ \\
$\mathrm{C} 191$ & $282.8 \pm 1.8$ & $5.94 \pm 0.82$ & $8.35 \pm 4.68$ \\
$\mathrm{C} 192$ & $281.7 \pm 1.5$ & $3.84 \pm 0.38$ & $7.97 \pm 2.21$ \\
\hline
\end{tabular}




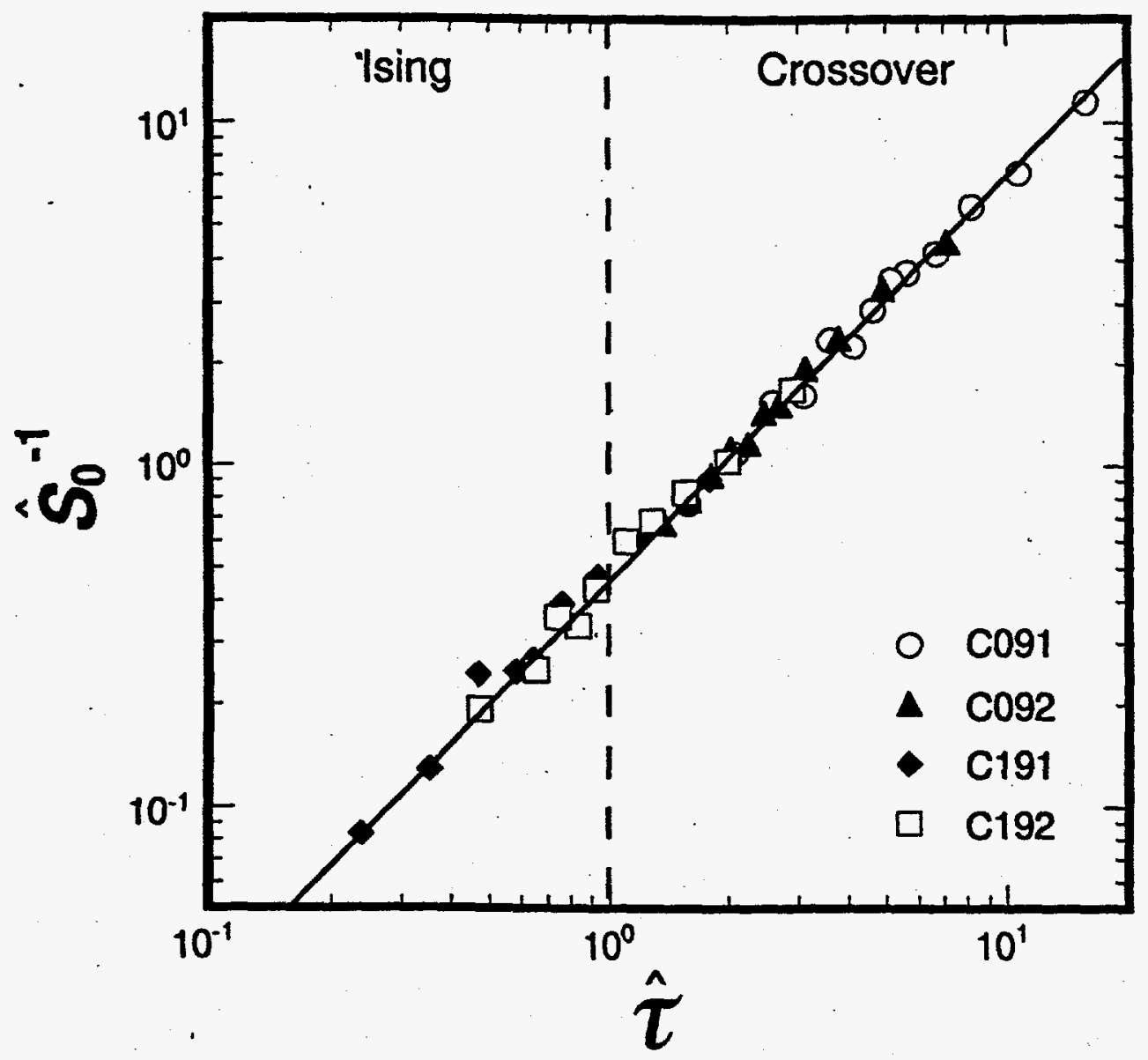

